\title{
Effect of energy losses and interactions during diffusive shock acceleration: applications to SNR, AGN and UHE Cosmic Rays
}

\author{
R.J. Protheroe \\ Department of Physics, The University of Adelaide \\ Adelaide, Australia 5005 \\ rprother@physics.adelaide.edu.au
}

\begin{abstract}
$\underline{\text { Abstract }}$
I discuss the shape of the high energy end of the spectrum of particles arising from diffusive shock acceleration in the presence of (i) additional diffusive escape from the accelerator, (ii) continuous energy losses, (iii) energy changes arising from interactions. The form of the spectrum near cut-off is sensitive to these processes as well as to the momentum-dependence of the diffusion coefficients and the compression ratio, and so the spectrum of any radiation emitted by the accelerated particles may reflect the physical conditions of the acceleration region. Results presented in this paper have applications in interpreting the spectral energy distributions of many types of astrophysical object including supernova remnants (SNR), active galactic nuclei (AGN) and acceleration sources of ultra high energy cosmic rays (UHE CR). Except for extremely nearby sources, spectral features imprinted on the spectrum of UHE CR during the acceleration process will be largely eroded during propagation, but the spectrum of UHE neutrinos produced in interactions of UHE CR with radiation, both during cosmic ray acceleration and subsequent propagation through the cosmic microwave background radiation, contains sufficient information to determine the cut-off momentum of the UHE CR just after acceleration for reasonable assumptions. Observation of these UHE neutrinos by the Pierre Auger Observatory may help in identifying the sources of the highest energy cosmic rays.
\end{abstract}

PACS Numbers: 98.70.Sa, 95.85.Ry, 98.38.Mz, 98.54.Gr

Keywords: cosmic rays, acceleration, propagation, neutrinos, supernova remnants, radio galaxies 


\section{Introduction}

For particle acceleration by electric fields induced by the motion of magnetic fields $B$ (including those at astrophysical shocks), the maximum rate of momentum gain by relativistic particles of charge $Z e$ can be written (in SI units)

$$
\left.\frac{d p}{d t}\right|_{\mathrm{acc}}=\xi(p) Z e c B
$$

where $\xi(p)<1$ and depends on the details of the acceleration mechanism. For example, in diffusive shock acceleration (DSA) $\xi(p)$ will depend on the shock velocity and diffusion coefficients. I shall refer to $\xi(p)$ as the acceleration rate parameter. Detailed and rigorous treatments of DSA are given in several review articles [1, 2, 3]; see particularly the review by Jones \& Ellison [4, on the plasma physics of shock acceleration, which also includes a brief historical review and refers to early work.

Protheroe and Stanev [5] proposed a box model of DSA which was able to reproduce the essential features of DSA in a simple leaky-box scenario. Particles were injected into the box at momentum $p_{0}$, and while inside the box their energy increased at the average rate that would apply given the physical parameters of the shock acceleration being modelled, i.e. the upstream and downstream diffusion coefficients, the shock velocity and compression ratio. Particles being accelerated leak out of the box, again at the average rate that would apply given the physical parameters, and these escaping particles represent the accelerated particles escaping downstream in the standard shock acceleration picture. This simple scheme is very easy to implement in a numerical or Monte Carlo program for investigating the structure of pile-ups and cut-offs in the case of adding additional leakage terms (e.g. simulating the finite size of any accelerator), energy losses during acceleration (e.g. synchrotron loss) or particle interactions such as inverse Compton (IC) losses in the Klein-Nishina regime. These processes could be important in determining the detailed shape of spectrum of non-thermal radiation from SNR or AGN.

Where protons are accelerated, interactions with magnetic fields, matter or radiation may determine where and how the spectrum cuts off. Of current interest is the hoped-for observation of $\pi^{0} \mathrm{TeV}$ gamma rays from SNR from $p p$ interactions of cosmic rays freshly accelerated at SNR shocks. At higher energies, the UHE CR may interact with background radiation fields, including the cosmic microwave background radiation (CMBR), by pion photoproduction interactions both during acceleration and propagation. This leads to a predicted spectral cut-off, the Greisen-Zatsepin-Kuzmin (GZK) cut-off [6, 7] at about $10^{20} \mathrm{eV}$ for particles which have travelled more than a few tens of Mpc from their sources. However, several experiments have reported UHE CR events with energies above $10^{20} \mathrm{eV}$

[8] with the highest energy event having $3 \times 10^{20} \mathrm{eV}$ [9], although the latest HiRes data [10] seems to show a cut-off consistent with the expected GZK cut-off.

Information about the acceleration environment is, in principle, imprinted in the shape of the high energy end of the spectrum of accelerated particles. In the case of the UHE CR, one of the main applications of the present work is to see to what extent this information 
remains, either in the cosmic ray spectrum, or in the spectrum of secondary particles such as neutrinos produced during both acceleration and propagation [11, 12, 13. Hence it is important to calculate these fluxes resulting from cascading in the CMBR. Spectral data at energies above $10^{18} \mathrm{eV}$ and directional results, notably from the AGASA project, are very suggestive of fascinating, unexpected physics [14. A new era in the field is beginning with the commissioning of the Pierre Auger Observatory [15] which comprises a pair of $3000 \mathrm{~km}^{2}$ arrays (one under construction in Mendoza Province, Argentina, and one planned for Utah, U.S.A.) employing both particle and optical detectors. The Auger Observatory will also have the capability of detecting UHE neutrinos which could carry significant information about the sites of acceleration (see refs. [16, 17] for recent review of UHE CR).

I shall first examine the case of continuous energy losses, then consider how the spectral features are altered for the case of interactions with various mean inelasticity values. As a specific example, I shall take the acceleration and propagation of the UHE CR, and briefly re-examine the likely acceleration sites of UHE CR. That will be followed by a description of Monte Carlo simulations of DSA using the box model and incorporating Bethe-Heitler pair production and pion photoproduction interactions in the CMBR; the results obtained being applicable also to other temperature blackbody fields with suitable scaling. Finally, I shall propagate these spectra to see whether any useful features remain in the UHE CR spectrum, and whether or not the spectrum of secondaries such as neutrinos can be used to infer physical conditions in the acceleration region of UHE CR.

\section{Roll-offs and cut-offs in the spectrum of acceler- ated particles}

The inclusion of escape processes, in addition to the usual escape downstream leads to a smooth roll-off in the spectrum of accelerated particles which is most pronounced at momenta near the momentum at which the two escape rates are equal, which I shall refer to as the "maximum momentum", $p_{\max }$. This smooth roll-off occurs over up to three decades in energy and the spectrum can extend beyond $p_{\max }$. Its shape depends on the momentum dependence of the diffusion coefficients, on the momentum dependence of the additional escape rate, and on the intrinsic differential spectral index, $\Gamma$, of the accelerated particles which would apply for the case of no energy losses or additional escape processes. Such roll-offs have very recently been noted in test-particle Monte Carlo simulations of shock acceleration at shocks in a cylindrical jet geometry where there is sideways leakage out of the jet [18.

When continuous energy losses are included the spectrum is cut-off sharply at a momentum at which the total rate of momentum change is zero, and I shall refer to this as the "cut-off momentum", $p_{\text {cut }}$. Depending on the intrinsic spectral index, and momentum dependence of the diffusion coefficient, a pile-up may occur before the cut-off. Protheroe and Stanev [5] subtracted from the momentum-gain rate a term representing the energy- 
loss rate. Drury et al. 19] showed that since the physical size of the "box" increases with energy, synchrotron losses can cause a particle in the downstream region to effectively "fall out" of the box, and that this process could be represented by an additional term in the formula for the escape rate. This term is included in the present work, and I confirm the result of Drury et al. 19] that the pile-up is less, particularly for low compression ratios.

In the box model of shock acceleration, particles of momentum $p_{0}$ are injected into the acceleration zone, or "box", and while inside the box are accelerated at a rate $r_{\text {acc }}(p)$ and escape from the box at a rate $r_{\text {esc }}(p)$. These two rates uniquely determine the spectrum of accelerated particles, i.e. those escaping from the box. The rate of change of momentum during shock acceleration in the presence of energy losses is

$$
p r_{\text {acc }}(p) \equiv p\left[r_{\text {gain }}(p)-r_{\text {loss }}(p)\right]=\left.\frac{d p}{d t}\right|_{\text {total }}=\left.\frac{d p}{d t}\right|_{\text {gain }}-\left.\frac{d p}{d t}\right|_{\text {loss }} .
$$

Note that, by definition, $r_{\text {gain }}\left(p_{\text {cut }}\right)=r_{\text {loss }}\left(p_{\text {cut }}\right)$.

The momentum gain and escape rates depends on the diffusion coefficients, which are usually assumed to have a power-law dependence on momentum, $\kappa(p) \propto p^{\delta}$, with the exponent depending on the nature of the turbulence present in the magnetic field: $\delta=1 / 3$ (Kolmogorov spectrum), $1 / 2$ (Kraichnan spectrum) or 1 (completely disordered field). When the diffusion coefficients upstream and downstream have the same power-law dependence on momentum, $\kappa_{1}(p)=K_{1} p^{\delta}$ and $\kappa_{2}(p)=K_{2} p^{\delta}$, the momentum gain rate is $r_{\text {gain }}(p) \propto p^{-\delta}$, giving

$$
r_{\text {acc }}(p)=r_{\text {loss }}\left(p_{\text {cut }}\right)\left(\frac{p}{p_{\text {cut }}}\right)^{-\delta}-r_{\text {loss }}(p) .
$$

In the case of $d p / d t \propto p^{2}$ losses, such as synchrotron loss, $r_{\text {loss }}(p) \propto p$ and one has

$$
r_{\text {acc }}(p)=r_{\text {loss }}\left(p_{\text {cut }}\right)\left[\left(\frac{p}{p_{\text {cut }}}\right)^{-\delta}-\frac{p}{p_{\text {cut }}}\right] .
$$

If there are no energy losses and no additional escape processes a power-law spectrum results, and the integral spectral index , $(\Gamma-1)$, equals the ratio of the escape rate to the acceleration rate. In this case, the escape rate representing escape downstream is $(\Gamma-1) r_{\text {loss }}\left(p_{\text {cut }}\right)\left(p / p_{\text {cut }}\right)^{-\delta}$. The acceleration zone extends distances $L_{1}(p)=\kappa_{1}(p) / u_{1}$ and $L_{2}(p)=\kappa_{2}(p) / u_{2}$ upstream and downstream from the shock, respectively. Adding an extra escape term $r_{\mathrm{esc}}^{\max }\left(p, p_{\max }\right)$ responsible for the "maximum momentum", and an escape term $r_{\text {esc }}^{\text {fallout }}(p)$ resulting from energy losses causing particles to fall out of the box gives the total escape rate

$$
r_{\text {esc }}(p)=(\Gamma-1) r_{\text {loss }}\left(p_{\text {cut }}\right)\left(\frac{p}{p_{\text {cut }}}\right)^{-\delta}+r_{\text {esc }}^{\max }\left(p, p_{\max }\right)+r_{\text {esc }}^{\text {fallout }}(p)
$$


For the case of a constant extra escape rate as adopted by [5]

$$
r_{\mathrm{esc}}^{\max }\left(p, p_{\max }\right)=(\Gamma-1) r_{\text {loss }}\left(p_{\text {cut }}\right)\left(\frac{p_{\text {max }}}{p_{\text {cut }}}\right)^{-\delta},
$$

and for the case of diffusive escape out of the box, e.g. diffusion perpendicular to the shock normal to the edge of the acceleration region with diffusion coefficient $\kappa \propto p^{\delta}$,

$$
r_{\text {esc }}^{\max }\left(p, p_{\max }\right)=(\Gamma-1) r_{\text {loss }}\left(p_{\text {cut }}\right)\left(\frac{p_{\max }^{2}}{p p_{\text {cut }}}\right)^{-\delta} .
$$

This latter case is probably more realistic than constant escape, and will give weaker roll-offs.

The escape rate resulting from energy losses is

$$
r_{\text {esc }}^{\text {fallout }}(p)=\left.\frac{1}{L(p)} \frac{d L_{2}}{d p} \frac{d p}{d t}\right|_{\text {loss }}
$$

where $L(p)=L_{1}(p)+L_{2}(p)$, and only the decrease of $L_{2}(p)$ with decreasing momentum contributes to escape as particles falling out of the upstream part of the box (length $L_{1}(p)$ ) will be advected back into the box. For $\kappa_{1}(p)=K_{1} p^{\delta}$ and $\kappa_{2}(p)=K_{2} p^{\delta}$,

$$
r_{\text {esc }}^{\text {fallout }}(p)=\delta \ell_{2} r_{\text {loss }}(p)
$$

where $\ell_{2} \equiv L_{2}(p) / L(p)$ is related to the compression ratio $R=u_{1} / u_{2}=(2+\Gamma) /(\Gamma-1)$,

$$
\ell_{2}=\frac{K_{2} R}{K_{1}+K_{2} R}=\frac{2+\Gamma}{\left(K_{1} / K_{2}\right)(\Gamma-1)+2+\Gamma} .
$$

Drury et al. [19] appear to use $K_{2}=K_{1}$ (possibly to represent a parallel shock for which in an ideal case $\left.B_{2}=B_{1}\right)$, giving $\ell_{2}=(2+\Gamma) /(1+2 \Gamma)=4 / 5$ for a strong shock. For a very weak shock $\ell_{2} \rightarrow 1 / 2$. For a perpendicular shock, for which in an ideal case $B_{2}=R B_{1}$, one might expect $K_{2}=K_{1} / R$, giving $\ell_{2}=1 / 2$. Except where otherwise stated, I shall use $\ell_{2}=1 / 2$ in this paper.

\section{Spectrum for cut-off due to continuous losses}

At time $t$ after injecting $N_{0}$ particles of momentum $p_{0}$ into the acceleration zone, the number of particles remaining in the acceleration zone, $N(t)$, is obtained by solving

$$
\frac{d N}{d t}=-N(t) r_{\mathrm{esc}}[p(t)]
$$

which has solution

$$
N(t)=N_{0} \exp \left[-\int_{0}^{t} r_{\mathrm{esc}}[p(t)] d t\right]=N_{0} \exp \left[-\int_{p_{0}}^{p} r_{\mathrm{esc}}(p) \frac{d t}{d p} d p\right] .
$$


Hence, the spectrum of escaping particles is

$$
F_{\mathrm{esc}}(p)=\frac{d N_{\mathrm{esc}}}{d p}=-\frac{d N}{d t} \frac{d t}{d p}=N_{0} \frac{r_{\mathrm{esc}}(p)}{p r_{\mathrm{acc}}(p)} \exp \left[-\int_{p_{0}}^{p} \frac{r_{\mathrm{esc}}(p)}{p r_{\mathrm{acc}}(p)} d p\right] .
$$

For continuous $d p / d t \propto p^{2}$ losses (synchrotron loss or IC scattering in the Thomson regime), and defining $x \equiv p / p_{\text {cut }}$, from Eq. 13] the spectrum for the case of $x_{0} \ll x<1 \ll$ $x_{\max }$ is given by

$$
F_{\mathrm{esc}}(x) \approx \frac{\delta \ell_{2} x+(\Gamma-1) x^{-\delta}}{x^{1-\delta}-x^{2}}\left(\frac{x}{x_{0}}\right)^{-(\Gamma-1)}\left(1-x^{1+\delta}\right)^{\left(\Gamma-1+\delta \ell_{2}\right) /(1+\delta)}
$$

For continuous $d p / d t \propto p$ losses (e.g., adiabatic), from Eq. 13 the spectrum for the case of $x_{\max } \gg 1$ is given by

$$
F_{\mathrm{esc}}(x) \approx \frac{\delta \ell_{2} x+(\Gamma-1) x^{-\delta}}{x^{1-\delta}-x}\left(\frac{x}{x_{0}}\right)^{\delta \ell_{2}}\left(\frac{x^{-\delta}-1}{x_{0}^{-\delta}-1}\right)^{(\Gamma-1) / \delta+\ell_{2}} .
$$

Note that in both cases, for $x \ll 1$ this reduces to $F_{\text {esc }}(p) \approx(\Gamma-1) x_{0}^{\Gamma-1} x^{-\Gamma}$, as it must. For $x_{\max } \rightarrow \infty$, and $\Gamma=1.5,2.0$ and 2.5 the shape of the cut-off is shown in Fig. 1. For synchrotron losses (Fig. 1a) the spectrum is hardly affected up to $x=0.3$ and strong pile-ups are present for $\Gamma \leq 2$, but for the case of adiabatic losses (Fig. 10) a pile-up occurs only for $\Gamma<2$ and $\delta=1$. Furthermore, adiabatic losses cause a much stronger turnover and affects the spectrum at significantly lower energies, down to $x \sim 10^{-3}$ for $\delta=1 / 3$. Of course the situation is modified for finite $x_{\max }$. In that case, the integral in Eq. 13 involves Hypergeometric Functions (as used in ref. [5]) and these are obtained by summation of an infinite series.

The spectral index as a function of momentum is given by

$$
\frac{d \ln F_{\mathrm{esc}}}{d \ln p}=\frac{p}{r_{\mathrm{esc}}(p)} \frac{d r_{\mathrm{esc}}}{d p}-\frac{p}{r_{\mathrm{acc}}(p)} \frac{d r_{\mathrm{acc}}}{d p}-1-\frac{r_{\mathrm{esc}}(p)}{r_{\mathrm{acc}}(p)} .
$$

One can obtain (the logarithm of) the differential energy spectrum by integrating Eq. 16 numerically from $\ln p_{0}$ to $\ln p$. For the case of inclusion of both synchrotron losses plus an extra momentum-dependent escape term (Eq. [7), Eq. 16] gives

$$
\begin{aligned}
\frac{d \ln F_{\mathrm{esc}}}{d \ln x}= & \frac{\delta \ell_{2} x+\delta(\Gamma-1)\left(x^{\delta} x_{\max }^{-2 \delta}-x^{-\delta}\right)}{\delta \ell_{2} x+(\Gamma-1)\left(x^{-\delta}+x^{\delta} x_{\max }^{-2 \delta}\right)}+\frac{\delta x^{-\delta}+x}{x^{-\delta}-x} \\
& -1-\frac{\delta \ell_{2} x+(\Gamma-1)\left(x^{-\delta}+x^{\delta} x_{\max }^{-2 \delta}\right)}{x^{-\delta}-x}
\end{aligned}
$$

and results for various $x_{\max }, \delta$ and $\Gamma$ values are shown in Fig. 2 These results apply equally to electrons and protons if synchrotron radiation is the dominant energy loss process. An application in which the cut-off in the proton energy spectrum is due to 
synchrotron loss is given by the Synchrotron Proton Blazar model for the jets of BL Lac type active galactic nuclei which have weak accretion disks [20, 21]. In contrast, in quasars with strong accretion disks, the spectrum of accelerated protons may be cut off by pion photoproduction interactions on thermal photons from the accretion disk. Cut-offs due to pion photoproduction will be discussed in Section 6 .

Finally, one can find the criterion for a pile-up by examining the spectral index as $x \rightarrow 1$ [19. The denominators of the 2 nd and 4 th terms of Eq. 17] tend to zero as $x \rightarrow 1$, and so the spectral index becomes infinite. The sign of the denominator is always positive if $\delta>-1$, and so whether the spectral index tends to $+\infty$ or $-\infty$ depends on the sign of the numerator. Hence a strong pile-up occurs if

$$
\delta\left(1-\ell_{2}\right)+2-\Gamma-(\Gamma-1) x_{\max }^{-2 \delta}>0 .
$$

Taking $x_{\max } \rightarrow \infty$ and $\ell_{2}=(2+\Gamma) /(1+2 \Gamma)$, as assumed by Drury et al. [19], I obtain

$$
(2-\delta)+(3+\delta) \Gamma-2 \Gamma^{2}>0
$$

from which the maximum values of $\Gamma$ for a pile-up are $2.069(\delta=1 / 3), 2.106(\delta=1 / 2)$, and $2.225(\delta=1)$. Taking the compression ratio to be $R=(2+\Gamma) /(\Gamma-1)$, for $\delta=1$ a pile-up is expected if $R>3.44949$ (in agreement with Drury et al. [19]). For $\ell_{2}=1 / 2$, as adopted in the present paper, the conditions for pile-ups for synchrotron losses and adiabatic losses are $\Gamma<2+\delta / 2$ and $\Gamma<1+\delta$, respectively, and are seen to be consistent with the spectra in Fig. 1,

\section{Spectrum for cut-off due to interactions}

Interactions of relativistic particles with matter or radiation generally result in the interacting particle losing energy, which is either given to the struck particle or photon (elastic collision) or used in production of secondary particles (inelastic collision). If the mean interaction length (mean free path) is $\lambda_{\text {int }}(p)$, for ultra-relativistic particles the rate of interaction is $r_{\text {int }}(p)=c / \lambda_{\text {int }}(p)$. If the mean inelasticity, i.e. average fraction of energy lost per interaction, is $\bar{\alpha}(p)$ then the effective loss rate for these non-continuous losses is $r_{\text {loss }}(p)=\bar{\alpha}(p) r_{\text {int }}(p)$. Of course, if $\bar{\alpha}(p) \ll 1$ then one can approximate the interactions as continuous energy losses. I shall now investigate how the spectrum of particles subject to interactions during acceleration differs from that of particles subject to continuous energy losses with the same effective loss rate.

In the simulation with $N_{0}$ particles injected, a particle is injected at time $t=0$ with momentum $p(0)=p_{0}=x_{0} p_{\text {cut }}$ and statistical weight $w(0)=w_{0}=1 / N_{0}$, and its subsequent momentum, $p(t)=x(t) p_{\text {cut }}$, and weight, $w(t)$, are determined after successive time steps $\Delta t$ chosen to be much smaller than the smallest time-scale in the problem; $\Delta t=0.01 x_{0}^{-\delta} / r_{\text {loss }}\left(p_{\text {cut }}\right)$ is used in these simulations. In each time step, first the momentum is changed, $p(t+\Delta t)=p(t)\left[1+\Delta t r_{\text {acc }}(p)\right]$, and then the probability of escaping in time $\Delta t$ is estimated as $P_{\text {esc }}=\left\{1-\exp \left[-\Delta t r_{\text {esc }}(p)\right]\right\}$ where $r_{\text {esc }}$ here does not include the 
$r_{\mathrm{esc}}^{\text {fallout }}$ term. Then $w(t) P_{\mathrm{esc}}$ particles with momenta $p$ are binned in a histogram of accelerated particles, and the particle's weight is changed to reflect the fraction not escaping, $w(t+\Delta t)=w(t)\left(1-P_{\text {esc }}\right)$. Next, the probability of interacting in time $\Delta t$ is estimated, $P_{\text {int }}=\left\{1-\exp \left[-\Delta t r_{\text {int }}(p)\right]\right\}$, and a random number is generated to determine whether or not an interaction takes place. If an interaction does take place, a random number is generated and used to sample the inelasticity $\alpha$, and the particle's momentum ( $p_{i}$ before the interaction) is changed to $p=p_{i}(1-\alpha)$. Now the additional escape resulting from the momentum decreasing during the interaction is considered. The probability of the particle immediately escaping downstream due to "falling out of the box" is estimated,

$$
\text { Prob. (escape, } \left.p_{i} \rightarrow p\right)=\frac{L_{2}\left(p_{i}\right)-L_{2}(p)}{L\left(p_{i}\right)}=\left[1-\left(\frac{p}{p_{i}}\right)^{\delta}\right] \ell_{2},
$$

and a random number is generated to determine if this happens. If the particle does escape, then $w(t+\Delta t)$ particles with momenta $p$ are binned in a histogram of accelerated particles, and a new particle is injected with momentum $p_{0}$ and weight $w_{0}$. If the particle does not escape, then the particle's momentum and weight are evolved through a new time step as described above.

The resulting spectra are given in Figs. 3 and 4 give results for $\delta=1 / 3$ and 1 for the cases for which we already have results for the corresponding continuous loss process, i.e. the same $r_{\text {loss }}(p)$ but with $\bar{\alpha} \rightarrow 0$, for $\bar{\alpha}=0.005,0.05$ and 0.5 , and (for simplicity) an inelasticity distribution which is constant for $0<\alpha \leq 2 \bar{\alpha}$ and zero elsewhere. In each figure I show spectra for combinations of $\Gamma=1.75,2$ and 2.25 , and $r_{\text {loss }}(p) \propto p^{0}, p^{0.5}$ and $p^{1}$. The histograms show Monte Carlo results for the three inelasticity values, and the solid curve gives the analytic result for continuous losses which is found to be in excellent agreement with the $\bar{\alpha}=0.005$ case. As is to be expected, where a strong pile-up (spike) is present for the case of continuous losses, it remains but is reduced if $\bar{\alpha}=0.05$, and is completely removed if $\bar{\alpha}=0.5$. In the last case, the spectrum generally continues to momenta above $p_{\text {cut }}$, and there can also be an enhancement (weak pile-up) at lower momenta.

The spectra obtained above for the $\bar{\alpha}=0.5$ and $r_{\text {loss }}(p) \propto p^{0}$ would be directly applicable to the case where proton acceleration is cut off as a result of hadronic interactions with matter above the threshold for multiple pion-production because the proton-nucleon inelastic cross section increases only logarithmically with energy, and may be approximated as constant over, say, one decade in energy. Furthermore, for this case a not-unreasonable approximation for the inelasticity distribution is a uniform distribution between 0 and 1. Although other processes are normally assumed to cut off the spectrum, if the matter density is sufficiently high, it is in principle possible for hadronic collisions of protons with matter to play this role. Another possible application, again for the $r_{\text {loss }}(p) \propto p^{0}$ case, would be for acceleration of electrons in relatively dense astrophysical matter in which relativistic bremsstrahlung could cut off the electron spectrum. Although a flat inelasticity distribution is not such a good approximation in this case, this process does 
involve discontinuous energy losses comparable to the electron's energy, and so the results for $\bar{\alpha}=0.5$ may serve as a guide.

Perhaps, the most interesting application, again for the $\bar{\alpha}=0.5$ and $r_{\text {loss }}(p) \propto p^{0}$ case would be for acceleration of high energy protons in a dense radiation field to energies well above the pion photoproduction threshold where $r_{\text {loss }}(p)$ is approximately constant and $\bar{\alpha}$ ranges from 0.2 to 0.5 depending on proton energy. Near threshold $r_{\text {loss }}(p)$ is strongly dependent on momentum, and just above threshold the momentum dependence changes smoothly and passes through $r_{\text {loss }}(p) \propto p$ and $r_{\text {loss }}(p) \propto p^{1 / 2}$ before becoming approximately constant well above threshold. For proton acceleration in a radiation field where pion photoproduction cuts off the spectrum, one can expect to see features of the type shown in Figs. 3 [ 4 depending on the cut-off momentum, and momentum dependence of the diffusion coefficient. This process will be discussed in greater detail in the next two sections for the case of black-body radiation.

\section{Cut-off momentum for acceleration in black body radiation: sites of $\mathrm{UHE} \mathrm{CR}$}

Although I shall concentrate here on cut-offs due hadronic interactions with photons of the CMBR during acceleration of UHE CR protons, the results presented will be equally applicable to proton acceleration in any black body radiation field provided the proton momenta and interaction time-scales are scaled appropriately. For example, they may be applicable to proton acceleration in quasars in the nearly black-body accretion disk radiation. For an arbitrary temperature $T=2.725 T_{2.7} \mathrm{~K}$, the momenta quoted should be replaced by $p / T_{2.7}$.

In the case of acceleration of UHE CR, interactions with the CMBR may be important if the magnetic field is less than $\sim 10^{-4} \mathrm{G}$. In this case, the gyroradii are rather large, and possible acceleration sites could be hot-spots in lobes of giant radio galaxies [22, 23], or MHD generated electric fields along the whole length of an AGN jet such as that of Cen A 24. Inevitably, acceleration times will be long, and one should also include redshift losses, and Bethe-Heitler pair production which has a somewhat lower threshold than pion photoproduction. In Bethe-Heitler pair production, the energy lost per interaction is small, less than $1 \%$, and so this process can be treated adequately as a continuous loss

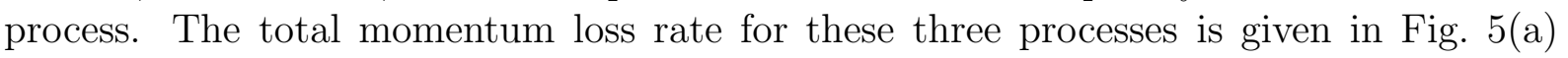
together with the momentum-gain rates required to reach various cut-off momenta for $\delta=1 / 3,1 / 2$ and 1 .

By plotting magnetic field vs. size of various astrophysical objects, Hillas 22] identified possible sites of acceleration of UHE CR based on whether or not the putative source could contain the gyroradius of the accelerated particles, and on the likely velocity of scattering centres in these sites (arguments similar to those that led to Eq. 11). Following Hillas [22] one finds that possible sites included neutron stars $\left(10^{7}-10^{13} \mathrm{G}\right)$, gamma ray bursts and active galactic nuclei $\left(10^{3}-10^{4} \mathrm{G}\right)$, and lobes of giant radio galaxies and galaxy clusters 
$\left(10^{-7}-10^{-5} \mathrm{G}\right)$. This identification of possible sources does not take account of energy losses (synchrotron) and interactions (Bethe-Heitler and pion photoproduction) which can cut off the spectrum, and so apply an additional constraint. In the same paper, Hillas [22] used this additional constraint to narrow the field of possible sources to radio galaxy lobes and galaxy clusters, and showed that to accelerate protons to $\sim 10^{20} \mathrm{eV}$ large regions $(\sim \mathrm{Mpc})$ of relatively low magnetic field (less than $\sim 10^{-4} \mathrm{G}$ ) are needed, ruling out high magnetic field regions for the origin of UHE CR. For the higher magnetic fields in this range the spectrum is cut-off by synchrotron loss, and would be as discussed in Section 3. Thus, one of the very few plausible acceleration sites of UHE CR may be associated with the radio lobes of powerful radio galaxies, either in the hot spots [23] or possibly the cocoon or jet [25].

To estimate cut-off momenta (or energy), one needs plausible values for the acceleration rate. The following values [26] for the acceleration rate parameter in Eq. 1] are used: maximum possible acceleration rate $\xi\left(p_{\text {cut }}\right)=1$, plausible acceleration at perpendicular shock with speed $0.1 c, \xi\left(p_{\text {cut }}\right) \approx 0.04$, and plausible acceleration at parallel shock with speed $0.1 c, \xi\left(p_{\text {cut }}\right) \approx 1.5 \times 10^{-4}$. The latter value is consistent with that of Biermann and Strittmatter [27] who considered DSA of protons in radiation and magnetic fields of active galactic nuclei. Assuming a Kolmogorov spectrum of turbulence, equation 6 of Biermann and Strittmatter 27] would give an acceleration rate parameter at the maximum energy of $\xi=0.08 \beta_{s}^{2} b$ where $\beta_{s} c$ is the shock velocity and $b$ is the ratio of turbulent to ambient magnetic energy density; for $\beta_{s}=0.1$ this gives $\xi\left(p_{\max }\right) \approx 8 \times 10^{-4} b$. Based on the total momentum loss rate given in Fig. 國(a), the proton cut-off momentum is plotted in Fig. [5(b) as a function of magnetic field of protons for three adopted $\xi$-values (chain lines are for constant Larmor radius as labelled). This plot, which is based on that in ref. [26], clearly shows that to accelerate protons to $\sim 10^{20} \mathrm{eV}$ large regions of relatively low magnetic field $\sim 10^{-7}-10^{-3} \mathrm{G}$ are needed, apparently ruling out high magnetic field regions for the origin of UHE CR (see also ref. [28]). One sees that, in principle, protons can be accelerated up to $\sim 5 \times 10^{22} \mathrm{eV}$ in Mpc scale region with $\sim 10^{-5} \mathrm{G}$.

Fig. [6 is the "Hillas plot" with constraints added corresponding to the three curves in Fig. (5), and where the chain lines give constant proton energy values as indicated; sources to the right of the solid curves being excluded. A possible exception to this is in the case of relativistically beamed sources (e.g. for AGN see ref. [29], and for GRB see ref. [30]) where neutrons emitted along the direction of relativistic motion can be Doppler boosted significantly in energy. Another possible exception is the case of so called "one-shot" mechanisms (e.g. [31, 32]) where a particle is accelerated by an electric field along a nearly straight path which is essentially parallel to the magnetic field such that curvature and synchrotron losses are negligible. Suggested sites include polarization electric fields arising in plasmoids injected into a neutron star's magnetosphere [33] and magnetic reconnection in the magnetosphere of accretion induced collapse pulsars [34]. Another possibility is plasma wakefield acceleration, i.e. acceleration by collective plasma waves, possibly in the atmosphere of a GRB, or "surf-riding" in the approximately forcefree fields of the relativistic wind of a newly born magnetar [35. In these cases it is 
unclear whether the requirements of negligible radiation losses can be met. I will next discuss in some detail shape of the spectrum, where the cut-off is due to photoproduction losses, for a large range of cut-off momenta.

\section{Spectrum for cut-off due to photoproduction loss}

Treating pion photoproduction as a continuous loss process, the escape rate and acceleration rate are given by

$$
\begin{aligned}
& r_{\text {esc }}(p)=(\Gamma-1) r_{\text {loss }}\left(p_{\text {cut }}\right)\left[\left(\frac{p}{p_{\text {cut }}}\right)^{-\delta}+\left(\frac{p_{\text {max }}}{p_{\text {cut }}}\right)^{-\delta}\right]+\delta \ell_{2} r_{\text {loss }}(p) \\
& r_{\text {acc }}(p)=r_{\text {loss }}\left(p_{\text {cut }}\right)\left(\frac{p}{p_{\text {cut }}}\right)^{-\delta}-r_{\text {loss }}(p)
\end{aligned}
$$

and the spectral index is

$$
\begin{aligned}
\frac{d \ln F_{\mathrm{esc}}}{d \ln p}= & \frac{\delta \ell_{2} R(p) q(p)-\delta(\Gamma-1) x^{-\delta}}{\delta \ell_{2} R(p)+(\Gamma-1)\left(x^{-\delta}+x_{\max }^{-\delta}\right)}+\frac{\delta x^{-\delta}+R(p) q(p)}{x^{-\delta}-R(p)} \\
& -1-\frac{\delta \ell_{2} R(p)+C\left(x^{-\delta}+x_{\max }^{-\delta}\right)}{x^{-\delta}-R(p)}
\end{aligned}
$$

where

$$
R(p)=\frac{r_{\text {loss }}(p)}{r_{\text {loss }}\left(p_{\text {cut }}\right)}, q(p)=\frac{d \ln \left(r_{\text {loss }}\right)}{d \ln (p)}
$$

By examining the gradient of the solid curve in Fig. 5(a) one sees that $q(p)$ has a strong peak at $\sim 10^{20} \mathrm{eV}$, and this will give rise to a strong pile-up in the spectrum if the cut-off momentum is close to $\sim 10^{20} \mathrm{eV} / \mathrm{c}$. The resulting spectra for the continuous loss approximation are shown in Fig. 7, and do indeed show this strong pile-up if $p_{\text {cut }} \sim$ $10^{20} \mathrm{eV} / \mathrm{c}$. Pile-ups also occur near other $p_{\text {cut }}$ values, particularly if $p_{\max } \gg p_{\text {cut }}$, and are strongest if $\delta=1$. However, when the constant escape term (corresponding to, e.g., escape due to finite size of accelerator) dominates escape near $p_{\text {cut }}$, then the resulting steepening in the spectrum is the prominent feature in all cases (except where $p_{\text {cut }} \sim 10^{20} \mathrm{eV} / \mathrm{c}$ when there is always a strong pile-up). Of course, the above treatment assumes Bethe-Heitler pair production and pion photoproduction to be continuous loss processes. While this is a reasonable approximation for Bethe-Heitler pair production, it certainly is not for pion photoproduction, and in this case the sharp cut-off and pile-up will be partly smeared. Monte Carlo simulations have been performed to see to what extent any pile-ups shown in Fig. 7 are washed out.

In the Monte Carlo simulations, redshifting and Bethe-Heitler pair production were treated as continuous loss processes, but hadronic collisions of protons and neutrons were 
treated by the Monte Carlo method using the SOPHIA event generator [36]. Neutrons produced, e.g. in $p \gamma \rightarrow n \pi^{+}$, may decay inside the box into protons which continue to be accelerated, or they may escape from the box to decay outside into cosmic rays depending on the neutron's Lorentz factor and the physical dimensions of the acceleration region. Since the simulations are for a given $p_{\text {cut }}, p_{\max }$ and $\delta$, and the size of the box depends on the (unknown) normalization of the diffusion coefficients, I have performed simulations separately for the two extreme cases. In the first case the cosmic ray injection spectrum is made up only of protons escaping from the box, whereas in the second case the cosmic ray injection spectrum is made up of all neutrons produced inside the box plus protons escaping from the box. High energy gamma rays and neutrinos also result from photoproduction during acceleration (see ref. 37] for acceleration of protons and production of neutrinos during acceleration in $\sim 10^{5} \mathrm{~K}$ blackbody radiation).

The Monte Carlo results for proton acceleration in a black body radiation field with $\Gamma=2$ and $T_{2.7} p_{\max } c=10^{25} \mathrm{eV}$ are shown in Fig. 8 , with the left column being for neutrons decaying inside the box, and the right column being for neutrons escaping from the box before decaying. Upper thin curves are for escaping protons, and lower thin curves are for neutrinos (all flavours) produced during acceleration. Comparing the $T_{2.7} p_{\max } c=10^{25} \mathrm{eV}$ case (right column in Fig. (7) with the case of neutron decay inside the box (left column in Fig. 8) confirms the presence of the general features expected from treating interactions as a continuous loss process. The sharp features of Fig. [7 are somewhat rounded, and the spectrum contains particles with momenta exceeding $p_{\text {cut }}$, but this is to be expected because of the distributions of inelasticity and interaction time.

If neutrons escape from the box before decaying, the protons from neutron decay will not be further accelerated and this explains why the spectra in this case are lower than the case of neutrons decaying inside the box. In some models, protons are trapped outside of the acceleration region but inside the astrophysical source, and so it is of interest to know separately the spectrum of escaping neutrons produced during the acceleration process (there will also be be a component due to pion photoproduction of the trapped accelerated protons). The contribution of escaping neutrons to the total escaping proton flux is show in Fig. 8 by the thick curves.

For the first two spectra, with $p_{\text {cut }} c=1-3 \times 10^{19} \mathrm{eV}$, the cut-off is due to BetheHeitler pair production, and so there is no significant neutrino or neutron component. If $p_{\text {cut }} c=1-3 \times 10^{20} \mathrm{eV}$, there will be a strong pile-up. For $p_{\text {cut }} c>3 \times 10^{20} \mathrm{eV}$, the nature of the spectrum above $3 \times 10^{20} \mathrm{eV}$ depends strongly on the momentum dependence of the diffusion coefficient. For $\kappa \propto p^{1 / 3}$, and neutrons escaping, the spectrum essentially breaks from $\sim p^{-2}$ below the pile-up feature, to $\sim p^{-4}-p^{-3}$ above the pile-up feature (corresponding to $p_{\text {cut }} c=10^{21}-10^{23} \mathrm{eV}$ ). If neutrons decay inside the box, the flux above the pile-up feature is higher and more curved. Nevertheless, for $p_{\text {cut }} c>3 \times 10^{20} \mathrm{eV}$ and with $\kappa \propto p^{1 / 3}$ the flux at $p \sim p_{\text {cut }}$ is substantially lower than expected without interactions. In other words, the effect of interactions cuts in at much lower momenta than $p_{\text {cut }}$, and this is most pronounced for $\kappa \propto p^{1 / 3}$. For $\kappa \propto p$ the spectra above $3 \times 10^{20} \mathrm{eV}$ generally continue as roughly the original $\sim p^{-2}$ power law and suffer an almost exponential cut-off 
at $p \sim p_{\text {cut }}$, with the cut-off occurring at a slightly lower momentum in the case of neutron escape. As would be expected, the spectra for $\kappa \propto p^{1 / 2}$ are intermediate between the the $p^{1 / 3}$ and $p^{1}$ cases.

\subsection{Spectrum after propagation}

Unless the source is very near by, and the propagation time is less than $\sim 10 \mathrm{Mpc} / \mathrm{c}$, then the observed spectrum will be severely affected by propagation. This is illustrated in Fig. 9] which shows spectra of protons and neutrinos (all flavours) escaping from the acceleration region (dotted curves) and after propagation for time $100 \mathrm{Mpc} / \mathrm{c}$ for the cases given in Fig. 8. Results (not shown) for $50 \mathrm{Mpc} / \mathrm{c}$ are only marginally different, implying that cascading is essentially complete by $100 \mathrm{Mpc} / \mathrm{c}$. The proton spectra after propagation for all cases are hardly distinguishable, as they all show, as expected, a prominent GZK cut-off at $10^{20} \mathrm{eV}$ with a pile-up just below the acceleration cut-off. Hence, it appears that one can conclude little about the acceleration cut-off energy and hence physical conditions of the acceleration region, by directly observing the spectrum of UHE CR.

Information is preserved in the neutrino spectrum, to some extent, about the proton cut-off energy and the momentum dependence of the diffusion coefficient during acceleration. During propagation, the energy of protons above the GZK cut-off is converted into secondary particles, including neutrinos which propagate essentially unhindered. As can be seen, the cut-off in the neutrino spectrum after propagation is directly related to the cut-off in the accelerated proton spectrum, and the spectral shape retains some information about the shape of the cut-off of the accelerated proton spectrum, and hence the physical conditions of the accelerator. The spectra plotted are effectively $E^{2} d N / d E$ and so are proportional to the energy flux of particles per logarithmic energy interval, or the "spectral energy distribution" (SED). In Fig. 10(a) I plot the energy at the peak of neutrino SED after propagation vs. proton acceleration cut-off momentum, and in Fig. 10)(b) the maximum neutrino energy, which I define here as the energy at which the neutrino $\mathrm{SED}$ is $1 / e$ of its peak value, vs. proton acceleration cut-off momentum. As can be seen, the maximum neutrino energy is a more useful measurement for determining the proton acceleration cut-off momentum as it is less subject to statistical fluctuations (in real or Monte Calo data) and is more sensitive to $p_{\text {cut }}$.

The inferred proton acceleration cut-off momentum is sensitive to whether or not the neutrons escape from the acceleration region, particularly for the case of a Kolmogorov spectrum of turbulence. However, one can still use Fig. 10(b) to put limits on the proton cut-off energy. For example, the observation of UHE neutrinos with $E_{\nu, \max }=3 \times 10^{20} \mathrm{eV}$ would imply a proton cut-off energy of $3-4 \times 10^{21} \mathrm{eV}(\delta=1), 5 \times 10^{21}-10^{22} \mathrm{eV}(\delta=1 / 2)$, and $1.5 \times 10^{22}-10^{23} \mathrm{eV}(\delta=1 / 3)$. 


\section{Conclusion}

In this paper I have discussed the shape of the high-energy part of the spectrum of particles accelerated by DSA in the presence of (i) additional diffusive escape from the accelerator, (ii) continuous energy losses, (iii) energy changes arising from interactions. I find that the shapes of spectral features (roll-offs, pile-ups, cut-offs) depend strongly on the momentum-dependence of the acceleration rate, which in turn depends on the momentum-dependence of the diffusion coefficient, $\kappa \propto p^{\delta}$, and on the physical process responsible for inhibiting acceleration. Hence, the shape of the high-energy part of the spectrum is important because it can give clues to physical conditions at the acceleration site. In some situations the accelerated spectrum could, in principle, be observed directly, but more usually the particle spectrum (if observed) would be affected by propagation from source to observer. Nevertheless, some features of the spectrum after acceleration could remain, or the spectrum of radiation emitted by the accelerated particles would retain some sensitivity to the features of the accelerated particle spectrum.

In the case of continuous losses where the spectrum is cut off at the momentum at which acceleration rate equals loss rate, pile-ups occur for $p^{2}$-losses for $\Gamma \leq 2$, and for $\Gamma=2.5$ if $\delta=1$. However, for $p^{1}$-losses, there is a fairly strong smooth cut-off except for the flattest spectrum considered, $\Gamma=1.5$ with $\delta=1$. In the case of additional escape processes inhibiting acceleration at momenta comparable to or below the cut-off momentum, no pile-ups are seen, and in all cases the spectra roll off smoothly at the momentum where the additional escape becomes comparable with the normal escape downstream. One application of this could be in detailed fitting the X-ray spectra of SNR [38, or other sources in which synchrotron radiation is observed. Another example where these subtleties could be important is in acceleration in AGN jets where, for some physical conditions, the spectrum could be cut-off either by adiabatic losses as the jet expands, or by leakage out of the jet. In the jet-frame a reasonable order of magnitude approximation for the time scales of both processes is the jet radius divided by $c$. Depending on which process dominates, and the physical parameters, the spectrum could be strongly cut off with spectrum affected well below the nominal cut-off momentum (adiabatic losses), or have a more gentle decline staring just before the nominal cut-off momentum and extending a bit above it (diffusive escape).

In the case of interactions, the fate of the spectral features depends largely on the mean inelasticity of the interaction. For very small inelasticity, the results are close to the equivalent continuous loss case, but if $\bar{\alpha} \sim 1$ pile-ups and cut-offs are smoothed and particle spectra extend beyond the nominal cut-off. I have considered in detail the case of pion photoproduction by protons on black body radiation, focusing on acceleration of UHE CR in the presence of the CMBR. Using plausible acceleration rate parameters, I have re-examined the physical conditions necessary to accelerate protons to UHE and, by considering a "Hillas plot" with constraints from photoproduction and synchrotron radiation added, confirm that the most likely of the originally proposed acceleration sites are in hot-spots in lobes of giant radio galaxies, as inferred by Hillas [22] and others 
subsequently. However, relativistically moving sources such as AGN jets and GRB do not need to accelerate particles directly to UHE as Doppler boosting from the plasma frame, in which the acceleration occurs, to the host-galaxy frame can increase the energies of escaping particles, for example, by 30 for AGN jets and 1000 for GRB. Thus, even though these sources appear to be ruled out on the modified Hillas plot, they could well be viable.

If the UHE CR are indeed accelerated, and do not result from a top-down scenario, then the high energy end of the accelerated particle spectrum could contain clues of the physical conditions of the acceleration region. The spectra on acceleration for different physical conditions are quite distinct. If the source of the UHE CR is Galactic, e.g. a GRB which occurred in our Galaxy in recent pre-historic times (say within a few tens of thousands of years), then one might directly observe pile-up features, possibly at superGZK energies. If the sources are extragalactic the particle spectrum one would observe would almost certainly have had all information about the part of the spectrum above $\sim 10^{20} \mathrm{eV}$, i.e. beyond the GZK cut-off, removed. However, UHE neutrinos produced during propagation can be used to estimate at least the cut-off energy of the protons just after acceleration, if some reasonable assumptions about momentum dependence of diffusion coefficients and size of the accelerator can be made. The neutrinos produced during propagation will not point back directly to the acceleration region, but will appear to come from a halo of size somewhere between the proton gyroradius in intergalactic space around the source and the pion photoproduction loss distance,i.e. $\sim 1$ to $15 \mathrm{Mpc}$, depending on the magnetic field environment around the source. At the $16 \mathrm{Mpc}$ distance of M87 this would mean neutrinos would arrive from this source at angles up to $4^{\circ}-$ $90^{\circ}$, whereas for Cen A at only $\sim 4 \mathrm{Mpc}$, one might directly observe cut-off features in the UHE CR spectrum, but one would observe only those neutrinos from the direction of Cen A produced during acceleration unless the accelerated cosmic rays are efficiently trapped near to Cen A. For more distant source candidates, it might be possible to identify some currently active sources of UHE CR. Finally, I mention that if heavy nuclei are accelerated then photodisintegration may take place during acceleration and as well as during subsequent propagation. Simulations are planned for this case and will be the subject of a future paper.

\section{Acknowledgments}

I thank Alina Donea and Greg Thornton for carefully reading the manuscript. This research is supported by a Discovery Project grant awarded by the Australian Research Council. 


\section{References}

[1] L.O'C. Drury, Space Sci. Rev. 36 (1983) 57

[2] R. Blandford, D. Eichler, Phys. Rep. 154 (1987) 1

[3] E.G. Berezhko, G.F. Krymski, Usp. Fiz. Nauk 154 (1988) 49

[4] F.C. Jones, D.C. Ellison, Space Sci. Rev. 58 (1991) 259

[5] R.J. Protheroe and T. Stanev, Astropart. Phys. 10 (1999) 185

[6] K. Greisen, Phys. Rev. Lett. 16 (1966) 748

[7] G.T. Zatsepin, V.A Kuzmin, Sov. Phys. JETP Lett. 4 (1966) 78

[8] M. Takeda et al., Astropart. Phys. 19 (2003) 447

[9] D.J. Bird et al., ApJ 441 (1995) 144

[10] T. Abu-Zayyad et al., Astropart. Phys., submitted astro-ph/0208301

[11] R.J. Protheroe and P.A. Johnson, Astroparticle Physics, 4, 253 (1996); erratum, 5, 215 (1996)

[12] T. Stanev, R. Engel, A. Mücke, R.J. Protheroe, J.P. Rachen, Phys. Rev. D 62 (2000) 093005

[13] K. Mannheim, R.J. Protheroe, and J.P. Rachen, Phys. Rev. D 63 (2001) 023003

[14] N. Hayashida et al., Astropart. Phys. 10 (1999) 303

[15] Auger Collaboration Contributions 2001, in Proceedings of XXVII Cosmic Ray Conference, Hamburg, Edited by M. Simon et al., Copernicus Gesellschaft 2001, Vol. 2, pp 699-787

[16] M. Nagano, A.A. Watson, Rev. Mod. Phys. 72 (2000) 689

[17] R.J. Protheroe and R.W. Clay, Pub. Astron. Soc. Austr. (2004), in press. astro-ph/0311466

[18] F. Casse, A. Markowith, Astron. Astrophys. 404 (2003) 405

[19] L.O'C. Drury, P. Duffy, D. Eichler, A. Mastichiadis, Astron. Astrophys. 347 (1999) 370

[20] A. Mücke, R.J. Protheroe, Astroparticle Physics 15 (2001) 121 
[21] A. Mücke, R.J. Protheroe, R. Engel, J.P. Rachen, T. Stanev, Astropart. Phys. 18 (2003) 593

[22] A.M. Hillas, Ann. Rev. Astron. Astrophys., 22 (1984) 425

[23] J.P. Rachen, P.L. Biermann, Astron. Astrophys. 272 (1993) 161

[24] R. Schopper, G. Thorsten Birk, H. Lesch, Astropart. Phys. 17 (2002) 347

[25] C.A. Norman, D.B. Melrose, A. Achterberg, ApJ 454 (1995) 60

[26] R.J. Protheroe, in "Topics in cosmic-ray astrophysics" ed. M.A. DuVernois, Nova Science Publishing: New York, 2000, pp 258-298

[27] P.L. Biermann,, \& P.A. Strittmatter, ApJ 322 (1987) 643

[28] M.V.Medvedev, Phy. Rev. E 67 (2003) 045401

[29] R.J. Protheroe, A.-C. Donea, A. Reimer, Astropart. Phys. 19 (2003) 559

[30] G. Pelletier, E. Kersalé, Astron. Astrophys. 361 (2000) 788

[31] C.A. Haswell, T. Tajima, J.-L. Sakai, ApJ 401 (1992) 495

[32] W.H. Sorrell, ApJ 323 (1987) 647

[33] C. Litwin, R. Rosner, Phys. Rev. Lett. 86 (2001) 4745

[34] E.M. de Gouvela Dal Pino, A. Lazarian, ApJ 560 (2001) 358

[35] J. Arons, Astrophysical Journal 589 (2003) 871

[36] A. Mücke, R. Engel, J.P. Rachen, R.J. Protheroe, T. Stanev, Comp. Phys. Com. 124 (2000) 290

[37] A.P. Szabo, R.J. Protheroe, Astropart. Phys. 2 (1994) 375

[38] A.-C. Donea, R.J. Protheroe, in preparation (2004) 



Figure 1: Differential energy spectra for $x_{0} \ll 1 \ll x_{\max }$ for $\ell_{2}=1 / 2, \delta$ as indicated, and $\Gamma=1.5$ (upper curves), 2.0 (middle curves) and 2.5 (lower curves). Results are shown for (a) $d p / d t \propto p^{2}$, (b) $d p / d t \propto p$. 

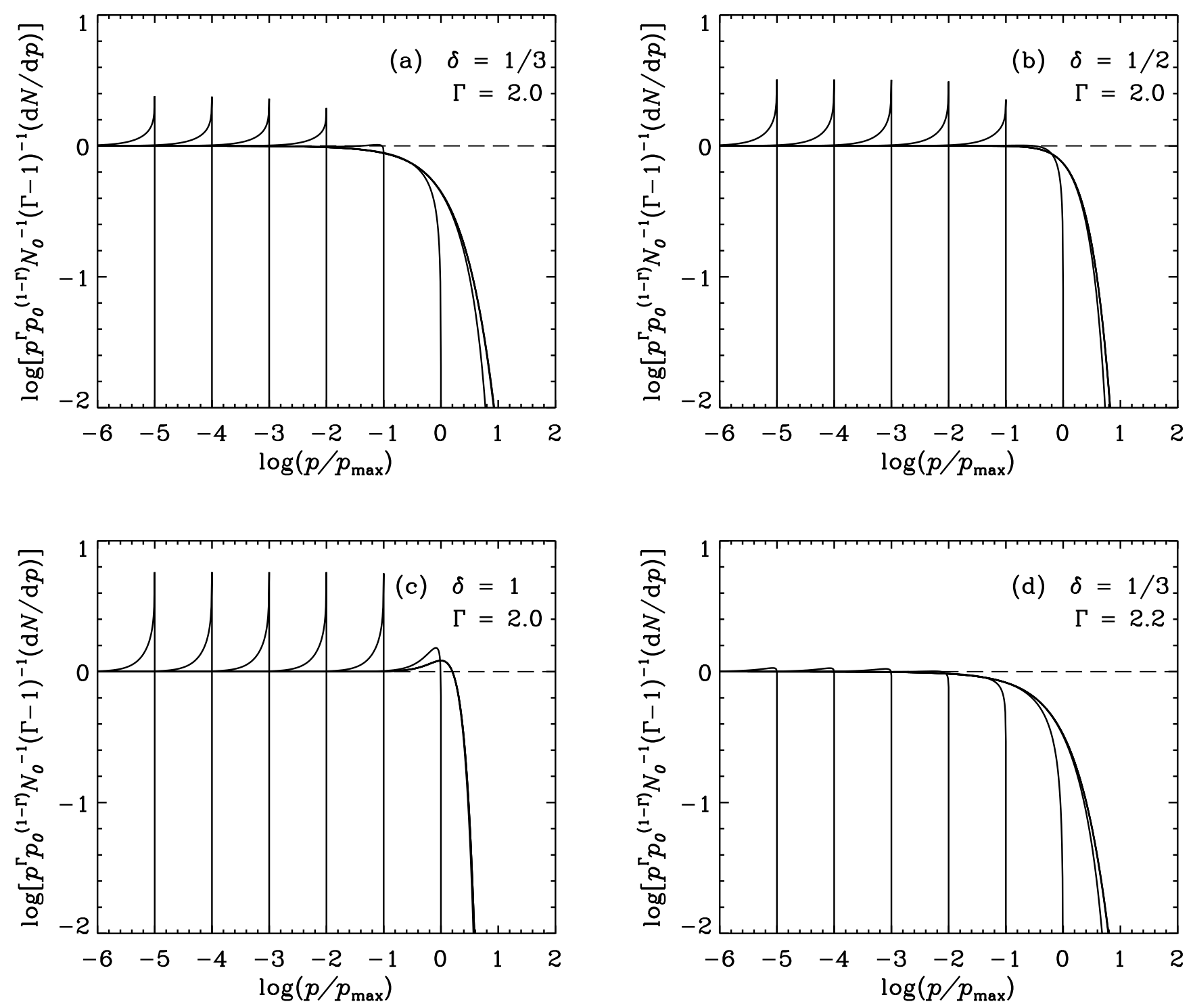

Figure 2: Differential energy energy spectrum for the case of $\Gamma=2$ for (a) $\delta=1 / 3$, (b) $\delta=1 / 2$, (c) $\delta=1$, and for (d) $\Gamma=2.2$ and $\delta=1 / 3$. In each case $\ell_{2}=1 / 2$, and results are shown for momentum-dependent escape at $p_{\max }$ and $p_{\text {cut }} / p_{\max }=10^{-5}$ (leftmost curve), $10^{-4}, \ldots, 10^{1}$ (rightmost curve). 


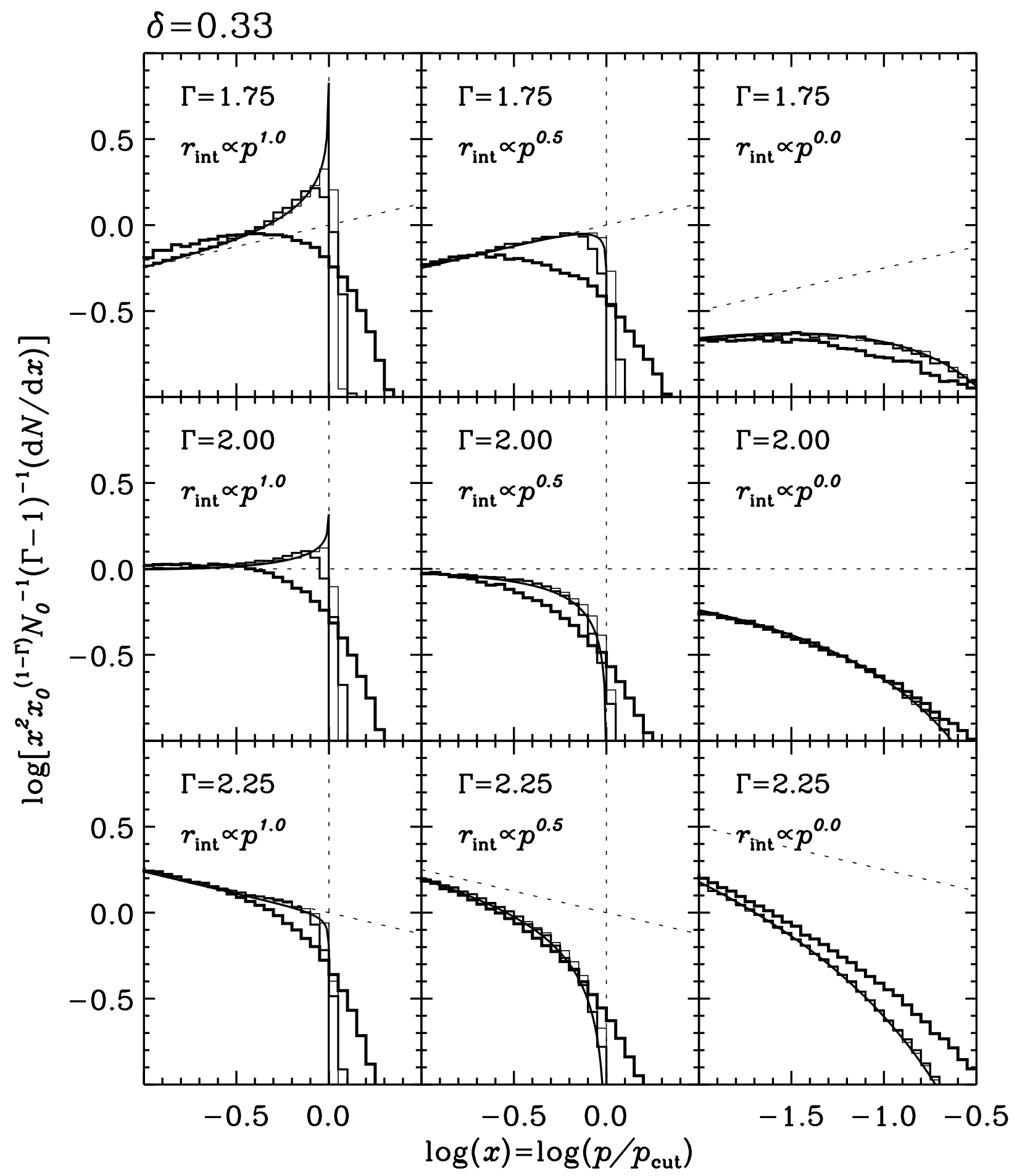

Figure 3: Spectra for $\delta=1 / 3, \ell_{2}=0.5$ and combinations of $\Gamma=1.75,2$ and 2.25, and $r_{\text {loss }}(p) \propto p^{0}, p^{0.5}$ and $p^{1}$ as indicated. In each case the histograms show Monte Carlo results for $\bar{\alpha}=0.005$ (thin histogram), $\bar{\alpha}=0.05$ (intermediate histogram) and $\bar{\alpha}=0.5$ (thick histogram), and the solid curve gives the analytic result for continuous losses. 




Figure 4: As Fig. [3 except $\delta=1$. 

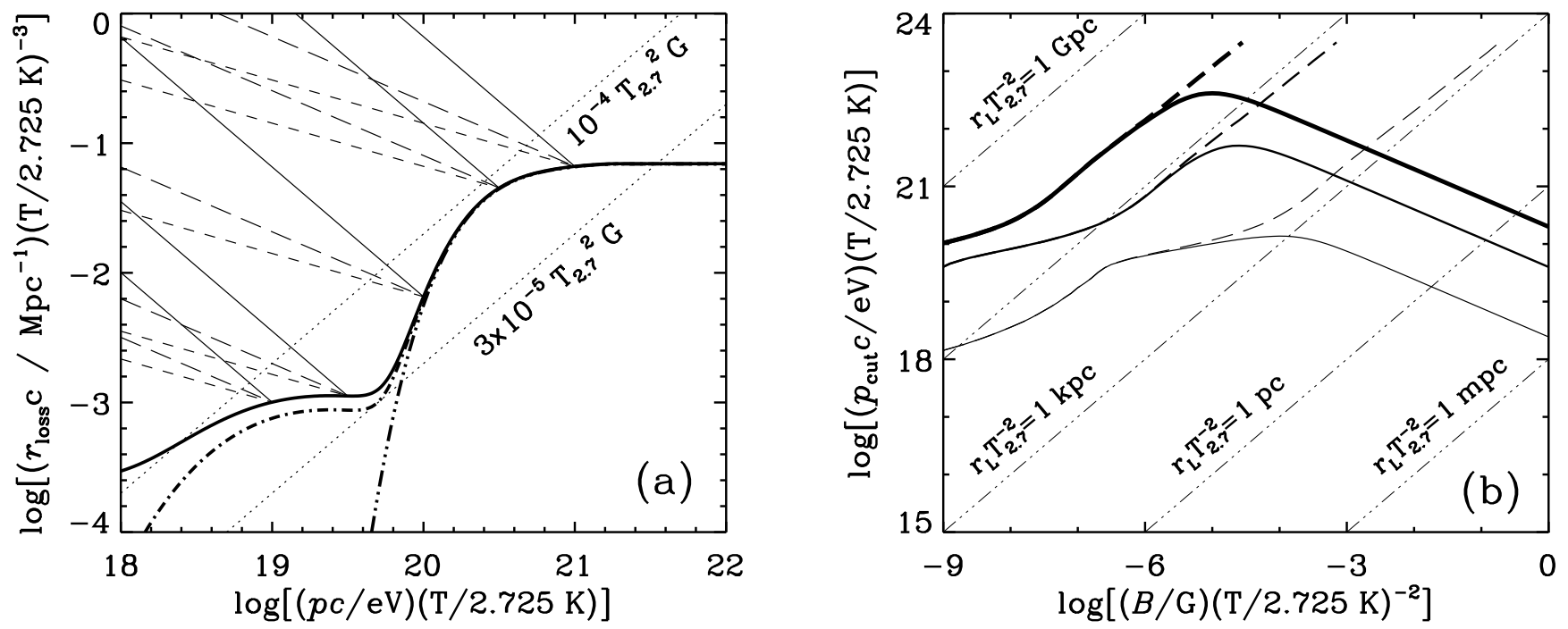

Figure 5: (a) Energy loss rate of protons $r_{\text {loss }}(p)$ in black body radiation of temperature $T=2.725 T_{2.7} \mathrm{~K}$ with losses by Bethe-Heitler pair production, pion photoproduction and redshifting [12. Note that redshift losses are included to illustrate their importance in the case of UHE CR acceleration in the $2.725 \mathrm{~K}$ CMBR. For other temperatures, the rate for Bethe-Heitler pair production plus pion photoproduction (chain curve) should be used; the dot-dot-dot-dash curve gives the loss rate for pion photoproduction only. Acceleration rates needed to reach cut-off momenta $p_{\text {cut }} c / T_{2.7}=10^{19}, 10^{19.5}, \ldots, 10^{21} \mathrm{eV}$ are shown by the thin lines: short dashed $(\delta=1 / 3)$, long dashed $(\delta=1 / 2)$ and solid $(\delta=1)$. Dotted lines show synchrotron loss rates for $B / T_{2.7}^{2}=3 \times 10^{-5} \mathrm{G}$ and $10^{-4} \mathrm{G}$. (b) Maximum energy as a function of magnetic field of protons for maximum possible acceleration rate $\xi=1$ (upper solid curve), plausible acceleration at perpendicular shock $\xi=0.04$ (middle solid curve), and plausible acceleration at parallel shock $\xi=1.5 \times 10^{-4}$ (lower solid curve). Dashed curves are limits from Bethe-Heitler pair production and pion photoproduction only (solid curves include synchrotron loss). Dot-dot-dot-dash curves are lines of constant Larmor radius as labelled. 




Figure 6: "Hillas plot" showing (chain curves) magnetic field vs. gyroradius for proton


such that the parameter space of accelerated particles is to the left of the curve corresponding to the chosen acceleration rate parameter. Typical size and magnetic field of possible acceleration sites (taken from Hillas [22]) are shown for neutron stars (ns), white dwarfs (wd), sunspots (ss), magnetic stars (ms), AGN (ag), interstellar space (is), supernova remnants (sn), radio galaxy lobes (rg), galactic disk (d) and halo (h), clusters of galaxies (cl) and intergalactic medium (ig). Typical jet-frame parameters of the synchrotron proton blazar model [21] and gamma ray burst model [30] are indicated by the open squares labelled "bl" and and "gb". 


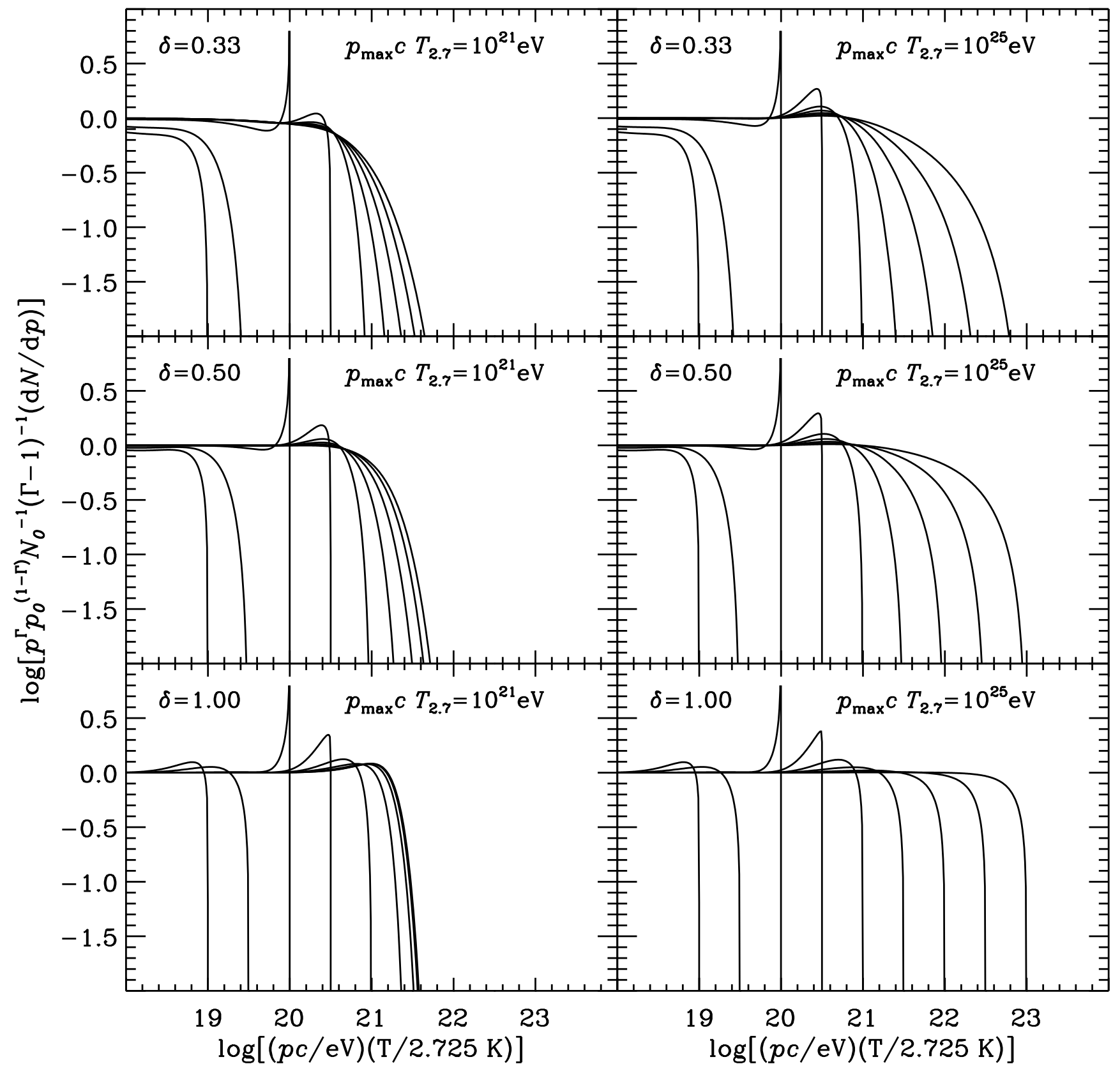

Figure 7: Differential momentum spectra of protons accelerated in the CMBR with continuous losses by redshifting, Bethe-Heitler pair production and pion photoproduction for $\Gamma=2$, and for $\delta$ and $p_{\max } c$ as indicated. In each case results are shown for $p_{\text {cut }} c=10^{19}$ (leftmost curve), $10^{19.5}, \ldots, 10^{23} \mathrm{eV}$ (rightmost curve). 


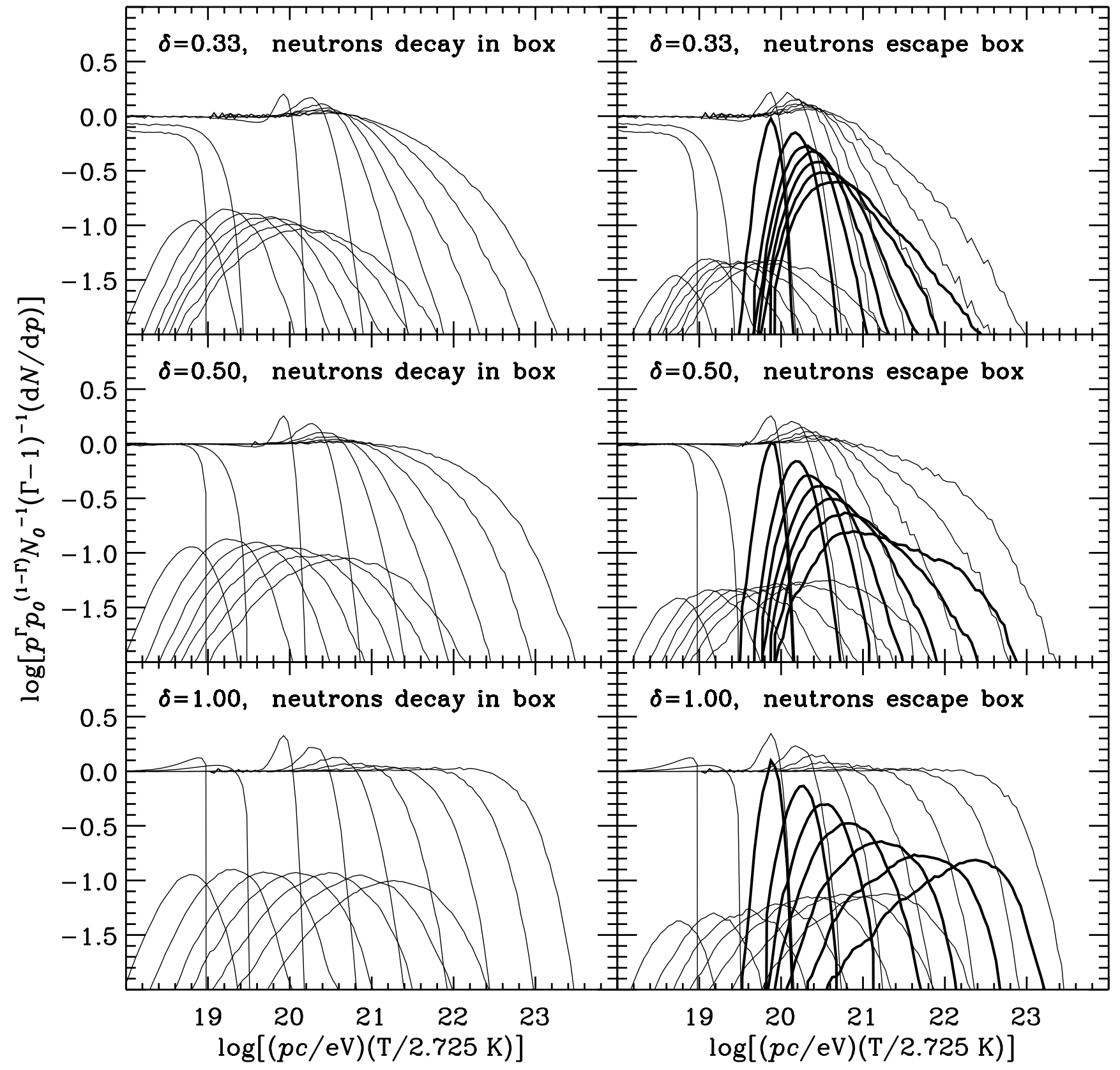

Figure 8: Differential momentum spectra of protons accelerated in the CMBR with continuous losses by Bethe-Heitler pair production and redshifting, and pion photoproduction interactions simulated using the SOPHIA event generator for $\Gamma=2, p_{\max } c=10^{25} \mathrm{eV}$, $\delta$ as indicated, and for neutron decay inside the box (left column) and neutron escape from the box (right column). Upper thin curves show the proton flux for $p_{\text {cut }} c=10^{19}$ (leftmost curve), $10^{19.5}, \ldots, 10^{23} \mathrm{eV}$ (rightmost curve). Lower thin curves total neutrino flux (all flavours), and thick curves show spectra of escaping neutrons, in both cases for $p_{\text {cut }} c=10^{20}$ (leftmost curve), $10^{20.5}, \ldots, 10^{23} \mathrm{eV}$ (rightmost curve). 




Figure 9: Spectra of protons and neutrinos (all flavours) escaping from the acceleration region (dotted curves) and after propagation for time $100 \mathrm{Mpc} / \mathrm{c}$ (solid curves) for $p_{\text {cut }} c=$ $10^{20}$ (leftmost curves), $10^{20.5}, \ldots, 10^{23} \mathrm{eV}$ (rightmost curves) for the cases given in Fig. 8 , 

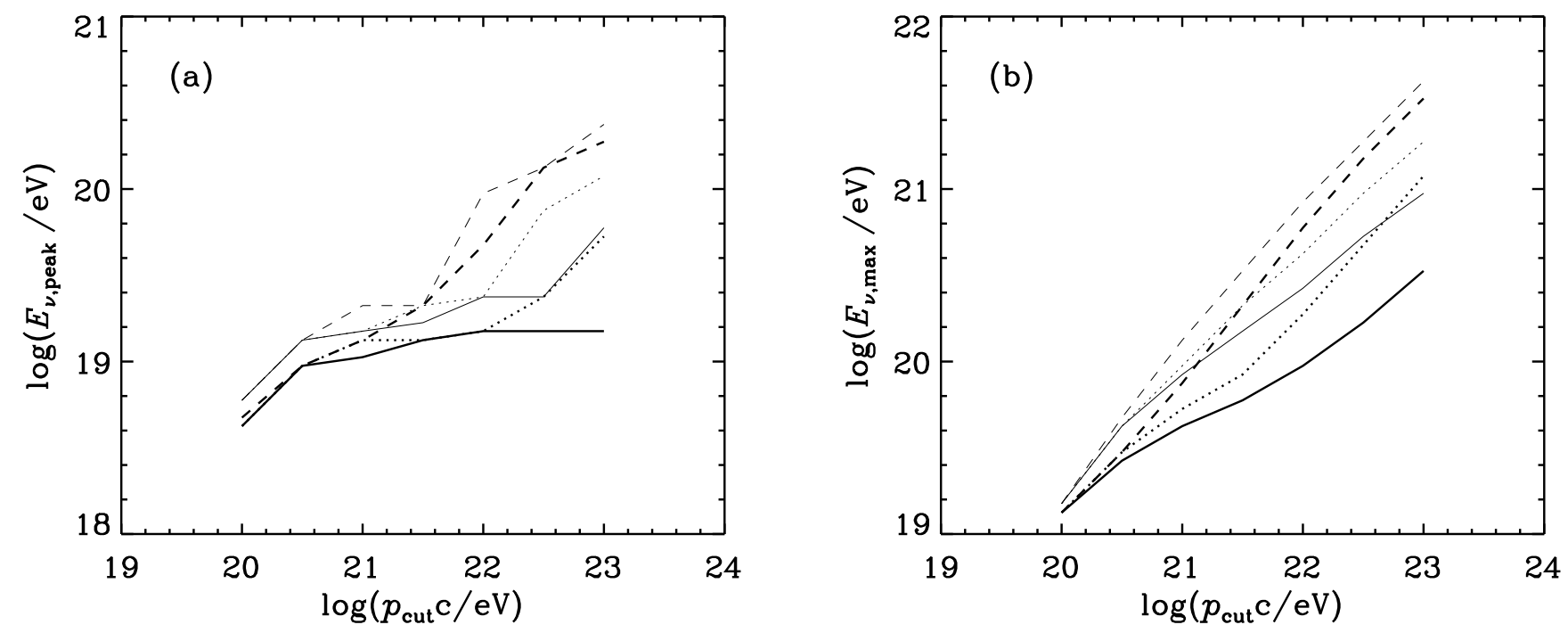

Figure 10: (a) Energy at peak of neutrino SED after propagation for time $100 \mathrm{Mpc} / \mathrm{c}$ vs. proton cut-off momentum, and (b) maximum neutrino energy defined as the energy at which the neutrino SED, after propagation for time $100 \mathrm{Mpc} / \mathrm{c}$, is $1 / e$ of its peak value vs. proton cut-off momentum. Thin curves are for neutron decay inside acceleration region, and thick curves are for neutron escape from acceleration region; results are shown for $\delta=1 / 3$ (solid curves), 1/2 (dotted curves) and 1 (dashed curves). 